\title{
Rotating Pan Coating Method
}

National Cancer Institute

\section{Source}

National Cancer Institute. Rotating Pan Coating Method. NCI Thesaurus. Code C112976.

A method that agitates a substrate in a rotating pan while it is sprayed with a layer of coating material. 